\title{
Conundrum of strongly coupled supercurrent flow in both under- and overdoped Bi-2212 round wires
}

\author{
Yavuz Oz $\odot,{ }^{1,2}$ Jianyi Jiang $\odot,{ }^{2}$ Maxime Matras $\odot,{ }^{2,3}$ Temidayo Abiola Oloye, ${ }^{2,3}$ Fumitake Kametani $\oplus^{2,3}$ \\ Eric E. Hellstrom, ${ }^{2,3}$ and David C. Larbalestier $\circledast^{2,3, *}$ \\ ${ }^{1}$ Department of Physics, Florida State University, 77 Chieftan Way, Tallahassee, Florida 32306, USA \\ ${ }^{2}$ Applied Superconductivity Center, National High Magnetic Field Laboratory, Florida State University, \\ 2031 East Paul Dirac Drive, Tallahassee, Florida 32310, USA \\ ${ }^{3}$ FAMU-FSU College of Engineering, Tallahassee, Florida 32310, USA
}

(Received 7 January 2021; revised 11 May 2021; accepted 25 May 2021; published 27 July 2021)

\begin{abstract}
$\mathrm{Bi}_{2} \mathrm{Sr}_{2} \mathrm{Ca}_{1} \mathrm{Cu}_{2} \mathrm{O}_{x}$ (Bi-2212) is the only high-temperature superconductor (HTS) available as a round wire with high critical current density $J_{c}$, which makes it a very compelling candidate for ultrahigh-field magnet applications. By contrast, other copper oxide HTS conductors like $R \mathrm{Ba}_{2} \mathrm{Cu}_{3} \mathrm{O}_{7-\delta}$ (where $R$ stands for rare earth) and $(\mathrm{Bi}, \mathrm{Pb})_{2} \mathrm{Sr}_{2} \mathrm{Ca}_{2} \mathrm{Cu}_{3} \mathrm{O}_{x}$ (Bi-2223) must be made in tape form to minimize the density of current blocking high-angle grain boundaries. Understanding the mechanism enabling high $J_{c}$ in round wire Bi-2212 is important intellectually because it breaks the paradigm that forces HTS conductors into tape geometries that reproduce their strong crystalline anisotropy. The biaxial growth texture of Bi-2212 developed during a partial melt heat treatment should favor high $J_{c}$, even though its $\sim 15^{\circ}$ full width at half maximum (FWHM) grain-to-grain misorientation is well beyond the commonly accepted strong-coupling range of $\leqslant 5^{\circ}$ misorientation. Its ability to be strongly overdoped should be valuable too since underdoped cuprate grain boundaries are widely believed to be weakly linked. Accordingly, we here study property changes after oxygen underdoping the optimized, overdoped wire. While $J_{c}$ and vortex pinning diminish significantly in underdoped wires, we were not able to develop the prominent weak-link signature [a hysteretic $J_{c}(H)$ characteristic] evident in even the very best Bi-2223 tapes with an $\sim 15^{\circ} \mathrm{FWHM}$ uniaxial texture. We attribute the high $J_{c}$ and lack of weak-link signature in our $\mathrm{Bi}-2212$ round wires to the high-aspect ratio, large-grain, basal-plane-faced grain morphology produced by partial-melt processing of Bi-2212. These features enable $c$-axis brick wall current flow when $a b$-plane transport is blocked. We conclude that the presently optimized biaxial texture of $\mathrm{Bi}-2212$ intrinsically constitutes a strongly coupled current path, regardless of its oxygen doping state.
\end{abstract}

DOI: 10.1103/PhysRevMaterials.5.074803

\section{INTRODUCTION}

Randomly oriented grain boundaries (GBs) are known to be a major impediment to the realization of high $J_{c}$ in polycrystalline high-temperature superconductors (HTSs) [1,2]. The most widely studied and clearly problematic GBs are planar [001] tilt GBs [1]. Dimos et al. [3,4] showed early on that $J_{c}$ across planar GBs decreases exponentially with an increasing grain-to-grain planar [001] tilt misorientation angle in $\mathrm{YBa}_{2} \mathrm{Cu}_{3} \mathrm{O}_{7-\delta}$ (YBCO), with the critical angle for the onset of weak-link behavior later shown to be as low as $2^{\circ}-3^{\circ}[5,6]$. The [001] tilt $\mathrm{GBs}$ in $\mathrm{Bi}_{2} \mathrm{Sr}_{2} \mathrm{Ca}_{1} \mathrm{Cu}_{2} \mathrm{O}_{x}$ (Bi2212) [7] and $(\mathrm{Bi}, \mathrm{Pb})_{2} \mathrm{Sr}_{2} \mathrm{Ca}_{2} \mathrm{Cu}_{3} \mathrm{O}_{x}(\mathrm{Bi}-2223)$ [8,9] display a similar dependence. Thus, the high- $J_{c}$ YBCO and $\mathrm{Bi}-2223$ anisotropic conductor forms needed for applications are made

\footnotetext{
*Corresponding author: larbalestier@asc.magnet.fsu.edu

Published by the American Physical Society under the terms of the Creative Commons Attribution 4.0 International license. Further distribution of this work must maintain attribution to the author(s) and the published article's title, journal citation, and DOI.
}

by complex and expensive fabrication processes to achieve the desired texture that minimizes the high-angle grain boundary density $[2,10]$. In marked contrast, recent work has developed $\mathrm{Bi}-2212$ into a high- $J_{c}$ round wire conductor $[2,10,11]$ without weak-link character and with architecture similar to low-temperature superconductor wires. The round wire form is very desirable for magnet applications because it allows the conductor to be produced in a low-hysteretic-loss, multifilamentary, twisted state in a macroscopically isotropic architecture that provides high magnetic field quality. The paradox of round wire $\mathrm{Bi}-2212$ is that it is now possible to make it with some 6 times higher $J_{c}$ [12-14] than carefully textured $\mathrm{Bi}-2223$ tapes $[15,16]$, a conundrum that seems to violate much of our hard-won understanding of HTS grain boundaries over the last 30 years.

A key step that enabled high $J_{c}$ in Bi-2212 was to fully densify filaments using an overpressure heat treatment, providing nearly perfect physical connectivity [2]. However, full densification does not provide a GB network without blocking misorientations or answer the question of how Bi-2212 is able to deliver much higher $J_{c}$ compared to uniaxially textured (stacks of grains with similarly oriented $c$ axes with a small average out-of-plane misorientation and essentially 
random in-plane misorientation) Bi-2223 tapes, in which a lengthy mechanical and thermal processing development only slowly led to practical $J_{c}$ values $[16,17]$. One part of the puzzle is provided by a recent comparison [10] of texture in high- $J_{c}$ Bi-2223 and $\mathrm{Bi}-2212$, which showed that a local biaxial texture (stacks of grains with minimal in-plane and out-of-plane misorientations that share a common [100] axis parallel to the filament axis and that have [010] and [001] axes that slowly rotate azimuthally along the filament axis) with a full width at half maximum (FWHM) of $\sim 15^{\circ}$ can be developed by first melting the Bi-2212 inside the $\sim 15 \mu \mathrm{m}$ diameter filaments and then allowing it to slowly solidify under sparse nucleation conditions that optimize rapid growth of long $a$-axis textured grains along the filament axis. Surprisingly, despite a $15^{\circ} \mathrm{FWHM}$ being well into the weak-link regime of [001] tilt YBCO bicrystals, round wire Bi-2212 can achieve a transport $J_{c}$ many times that of the state-of-the-art $\sim 15^{\circ}$ FWHM uniaxially textured Bi-2223 tape [15]. Moreover, the Bi-2212 wires lack the hysteretic field-increasing, field-decreasing, weak-link signature displayed by $\mathrm{Bi}-2223$ [10]. Indeed, a recent study found evidence of well-connected grains in an untextured bulk Bi-2212 sample but not in a similarly prepared Bi-2223 bulk sample [18]. This paradox of greatly divergent connectivity between two very similar siblings is what has stimulated the present study. We know that round wire $\mathrm{Bi}-2212$ shows great promise for ultrahighfield solenoids [2,19], for plasma fusion use [20], and for accelerator dipoles [12]. All such applications are made more attractive by higher $J_{c}$ values, and because we conclude that $J_{c}$ of the best present $\mathrm{Bi}-2212[13,14]$ is still less than $1 \%$ of Bi-2212's depairing current density [18,21], it seems clear that the active superconducting cross section is significantly less than unity. Understanding where obstacles to current flow occur and what they are is key to further enhancing $J_{c}$. We note that a recent change in $\mathrm{Bi}-2212$ powder type to one of more uniform and finer particle size has enabled a doubling of the maximum $J_{c}$ compared to the already good powder made by Nexans used in the wires in the present study [12-14].

All models of current flow in HTS compounds emphasize the primacy of $a b$-plane current flow, considering other possible current paths only when this path is blocked. Two prominent models for current transport emerged from early studies of the grain morphology of Bi-2223 tapes. The brick wall (BW) model [22,23] proposed that [001] $c$-axis twist GBs provide a strongly linked $c$-axis supercurrent path when the $a b$ plane was blocked as a result of the large basalplane-faced GB surface area provided by high aspect ratio grains. In contrast, the railway switch model [24,25] proposed that the $a b$-plane supercurrent path connects largely by low-angle, obliquely intersecting strongly coupled GBs called small-angle $c$-axis tilt grain boundaries. Steadily improving texture was then thought to minimize the need to access the intrinsically lower $J_{c} c$-axis paths between the dominant $a b$ plane paths, allowing an increase in overall $J_{c}$ of Bi-2223 tapes by better occupancy of the tape cross section by the transport current. Note that both models predict intra- and intergrain $c$-axis transport is a factor limiting the macroscopic $J_{c}$. The much lower magnitude of $c$-axis $J_{c}$ is at least partially compensated by the large area of the basal-plane-faced $c$-axis contacts in the BW case. Indeed, an earlier study by Cai et al. [26] found direct evidence for $c$-axis current paths in high- $J_{c}$ microsections of individual Bi-2223 filaments. In the context of the brick wall and railway switch models, Kametani et al. [10] offered possible explanations for the high $J_{c}$ and scarcity of weak links in $\mathrm{Bi}-2212$ round wires, despite their comparatively high $15^{\circ}$ FWHM GB misorientation, by focusing on particular differences in the Bi-2223 and Bi-2212 grain morphologies. Although both compound wires share a colony grain structure of stacked grains with a common $c$ axis, large [001] twist misorientations are much more common in $\mathrm{Bi}-2223$ than in $\mathrm{Bi}-2212$, as are $c$-axis tilts. $\mathrm{Bi}-2212$ has a much more ordered colony structure arising from the sparse grain nucleation and rapid growth of $a$-axis-oriented grains along the filament axis, which consume slower-growing, less favorably oriented grains on cooling from the liquid state. The result is a strong [100] $a$-axis texture with [010] at right angles and a slowly rotating [001] direction along the filament. In a conductor with several hundred such filaments, the overall effect is to produce a macroscopically isotropic behavior, even though each grain retains its full intrinsic electronic anisotropy. Because this Bi-2212 grain structure develops slowly from the melt, a much higher aspect ratio brick wall with much larger grains can form than in Bi-2223, where only a marginal liquid is present and the reaction to form $\mathrm{Bi}-2223$ occurs largely in the solid state. An additional nontrivial factor favoring higher $J_{c}$ in Bi-2212 is that, unlike Bi-2223 and YBCO, which can only be marginally overdoped, Bi-2212 can be strongly overdoped [11,27], potentially improving its GB connectivity since GBs in cuprates are widely believed to be underdoped with respect to the grains $[11,28]$. Overdoping also enhances intragrain vortex pinning by reducing electromagnetic anisotropy $\gamma$ [11,27,29-38], which improves vortex stiffness, helps grain-to-grain coupling [34,38], and increases the irreversibility field $H_{i r r}$, all of which together enhance $J_{c}[11,29-35]$. In summary one can clearly see many similarities between $\mathrm{Bi}-2223$ and $\mathrm{Bi}-2212$, but what matters today is to clearly distinguish the important differences that actually make 6 times higher overall $J_{c}$ possible in Bi-2212, whether by better connectivity or by better vortex pinning or some combination of the two. Technologically, it is a great advantage of Bi-2212 that very high $J_{c}$ is now possible in the desirable round wire, isotropic architecture preferred for conductor use, especially one with great architectural flexibility with respect to filament number, arrangement, and size.

Here we focus on one key attribute of Bi-2212, namely, its very broad doping range. Presently, optimized Bi-2212 is significantly overdoped, which is expected to compensate for any GB oxygen deficiency compared to the grains. With the expectation that global underdoping treatments would remove this compensation, we here investigate how optimized high- $J_{c}$, oxygen-overdoped $\mathrm{Bi}-2212$ round wires behave when systematically underdoped to better understand the extent to which oxygen doping affects GB connectivity, vortex pinning, and transport $J_{c}$. Previous studies on Bi-2212 have reported increased $H_{i r r}$ in Bi-2212 tapes [29,34], single crystals [31,32,35], and round wires [11,39]; improved $J_{c}(H)$ [11,29-31,33-35]; and changes in critical temperature $T_{c}[11,27,29,30,33,35-37,40]$ with increasing oxygen doping 
(a)

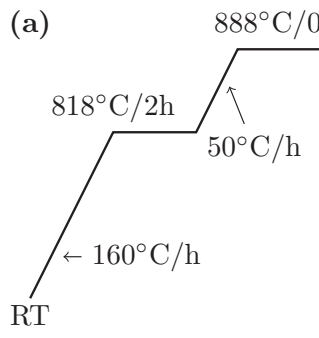

\section{$/ 0.2 \mathrm{~h}$}

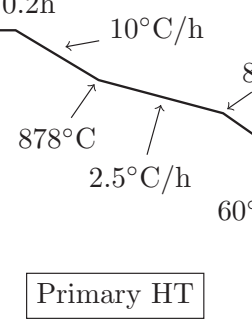

(b)

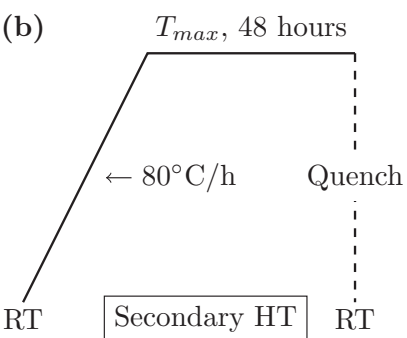

FIG. 1. (a) The primary and (b) secondary heat treatment procedures used to process the samples. Primary HT was carried out at 100 bars, whereas the secondary HT was carried out at 1 bar.

level. For the present study, as illustrated in Fig. 1, optimally overdoped (with respect to $J_{c}$ ) and full-density, overpressureprocessed $\mathrm{Bi}-2212$ round wires originally processed at $100 \mathrm{bar}$ total pressure (with 1 bar $\mathrm{O}_{2}$, balance $\mathrm{Ar}$ ) were each subjected to a secondary heat treatment (HT) at a low-oxygen partial pressure $\left(\mathrm{O}_{2}\right)$ to progressively underdope them, with the expectation that higher secondary heat treatment temperature would result in lower oxygen content in both grains and grain boundaries.

\section{METHODOLOGY}

\section{A. Sample preparation}

The Ag-Mg sheathed multifilamentary Bi-2212 round wires contained 18 bundles of 37 filaments $(37 \times 18)$, with as-drawn filament and wire diameters of $\sim 15 \mu \mathrm{m} / 0.8 \mathrm{~mm}$. They were fabricated by Oxford Superconducting Technology using the powder-in-tube technique with Nexans granulate $\mathrm{Bi}$ 2212 powder manufactured in 2013. Although state of the art at the time, this powder has since been substantially bettered by finer and more uniform powder manufactured by EngiMat that allows 2 times higher $J_{c}$ [14]. Approximately $1.2 \mathrm{~m}$ long pieces were melt processed under 100 bars $\left(1\right.$ bar $\mathrm{O}_{2}$, balance Ar) using the heat treatment profile in Fig. 1(a), and small pieces were then underdoped using the secondary heat treatment shown in Fig. 1(b), in a flowing gas, quench furnace under a 1 bar mixture of $\mathrm{Ar}-\mathrm{O}_{2}$ with a $\mathrm{pO}_{2}$ of 0.01 bar. The maximum temperature $T_{\max }$ was varied between $400{ }^{\circ} \mathrm{C}$ and $800^{\circ} \mathrm{C}$ to change the doping state, with increasing $T_{\max }$ values leading to lower oxygen content. The furnace was ramped at a constant rate of $80{ }^{\circ} \mathrm{C}$ per hour from room temperature $\left(\sim 30{ }^{\circ} \mathrm{C}\right.$ ) to $T_{\max }$ and equilibrated at $T_{\max }$ for $48 \mathrm{~h}$, after which the sample was pulled out of the hot zone to lock in the doping state.

Samples were prepared for electron backscatter diffraction (EBSD) orientation imaging microscopy by polishing to expose the longitudinal cross section of $\mathrm{Bi}-2212$ wires with diamond films of decreasing grit sizes, followed by ion polishing in a JEOL Cross Section Polisher using an Ar beam at $6 \mathrm{kV}$ and, finally, surface cleaning at $2 \mathrm{kV}$.

\section{B. Measurements and data analysis}

Standard four-point transport measurements of the critical current $I_{c}$ were conducted at field values ranging from 1 to $15 \mathrm{~T}$ in a superconducting magnet using a criterion of $1 \mu \mathrm{V} / \mathrm{cm}$. The field, applied perpendicular to the direction of current flow, was cycled in $1 \mathrm{~T}$ increments from 1 to $15 \mathrm{~T}$ and then back to $1 \mathrm{~T}$ to check for hysteresis in the $I_{c}(H)$ curves, with $I_{c}$ being measured at each field step. Conversion to $J_{c}$ was made using the fully dense $\mathrm{Bi}-2212$ areas measured by optical image processing after a densification heat treatment at $820^{\circ} \mathrm{C}$ [41]. Filaments were observed to be $60 \%-70 \%$ dense before reaction and greater than $95 \%$ dense after melt processing.

To compare the doping state of the samples, $T_{c}$ values were derived from measurements of magnetic moment as a function of temperature. The samples were zero field cooled to $4.2 \mathrm{~K}$ in a superconducting quantum interference device (SQUID) magnetometer and then warmed up to $100 \mathrm{~K}$ at $0.25 \mathrm{~K} / \mathrm{min}$ in a $2 \mathrm{mT}$ field applied perpendicular to the wire axis. $T_{c}$ was defined as the linear extrapolation of the $m(H)$ data to $m=0$ just before the sample fully transitioned to the normal state.

Magnetization hysteresis measurements were made at 4.2 and $20 \mathrm{~K}$ in a $14 \mathrm{~T}$ vibrating sample magnetometer with the wire axis perpendicular to the field. The magnetization loops $m(H)$ were run from -2 to 14 to $-2 \mathrm{~T}$ at a constant rate of $10 \mathrm{mT} / \mathrm{s}$, and magnetization hysteresis $\Delta M$ values were extracted, where $M$ is the sample magnetic moment normalized to the superconducting $\mathrm{Bi}-2212$ volume and $\Delta M$ is defined as $M$ (field decreasing) $M$ (field increasing). The Kramer field $H_{k}$ values were used to estimate $H_{i r r}$, the field at which $J_{c}$ drops to zero, by linear extrapolation of the $\left(\mu_{0} H\right)^{1 / 4}(\Delta m)^{1 / 2}$ versus field data at $20 \mathrm{~K}$ to the $H$ axis to define $H_{k}$ [42].

EBSD imaging was performed on the sample at a $70^{\circ}$ angle tilt using a $20 \mathrm{kV}$ electron beam in a Thermo Fisher Scientific Helios G4 high-resolution scanning electron microscope. EBSD data acquisition and processing were performed using the Aztec Nanoanalysis EBSD suite.

\section{FINDINGS}

Figure 2(a) shows changes in the $T_{c}$ transitions as a function of the secondary heat treatment $T_{\max }$. We note that the initial onset $T_{c}$ is below $80 \mathrm{~K}$ and clearly overdoped, and $T_{c}$ rises steadily to over $90 \mathrm{~K}$ with underdoping, before dropping again as the underdoping increases. The broad magnetization transitions are typical of $\mathrm{Bi}-2212$, where the filaments are electromagnetically decoupled from each other and the filament diameter is small. Extrapolating the hightemperature portion of the curve to zero magnetization allows us to define an upper limit $T_{c}$, shown in Fig. 2(b). $T_{c}$ clearly follows the parabolic trend typical of the relationship between $T_{c}$ and oxygen/carrier content in Bi-2212 [37,40], in agreement with the expectation that increasing $T_{\max }$ leads to 

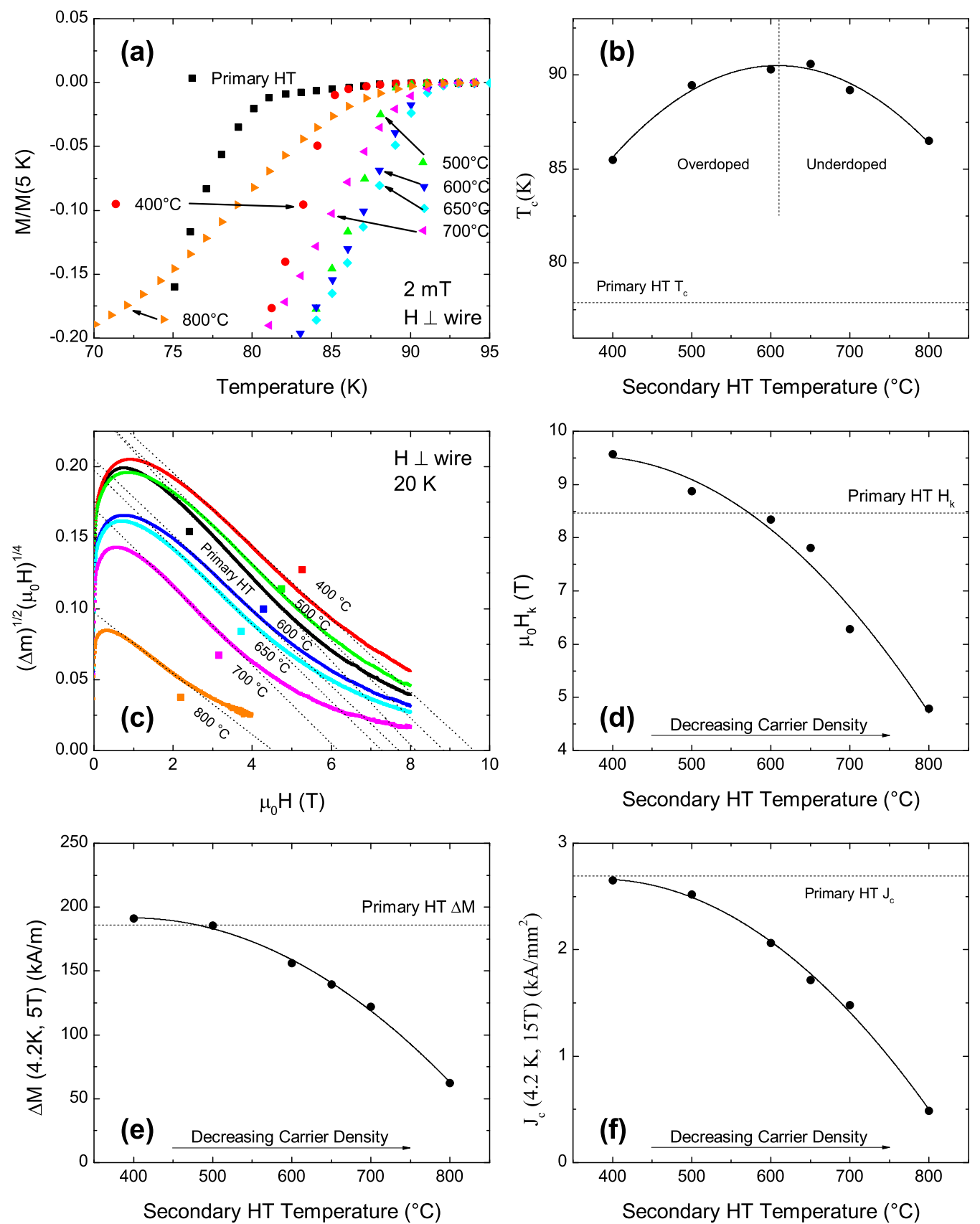

FIG. 2. (a) Zero field cooled (4.2 K) magnetization, measured in a SQUID magnetometer with $H=2 \mathrm{mT}$ applied perpendicular to the wire axis. As is typical for largely decoupled $\mathrm{Bi}-2212$ filaments, the transitions are all broad. (b) $T_{c}$ versus secondary heat treatment temperature $T_{\max }$. As $T_{\max }$ increased, the samples transitioned from the initially overdoped state to the underdoped state, reducing the carrier density. (c) Kramer function calculated from $\Delta m$ measurements. The linear regime after the initial peak can be extrapolated to zero to obtain the Kramer field $H_{k}$. (d) Kramer field, a measure of $H_{i r r}$, at $20 \mathrm{~K}$ versus $T_{\max }$. Vortex pinning, as judged by $H_{i r r}$, decreased with the decreasing carrier density induced by increasing $T_{\max }$. (e) Magnetization hysteresis $\Delta M$ (at $5 \mathrm{~T}, 4.2 \mathrm{~K}$ ) versus $T_{\max } . \Delta M$ decreased as the samples were increasingly underdoped, much like transport $J_{c}$. (f) Transport $J_{c}$ at $15 \mathrm{~T}, 4.2 \mathrm{~K}$ versus $T_{\max }$. Consistent with $\Delta M$ (e), transport $J_{c}$ went down as charge carriers were depleted. The horizontal dashed lines denote the value for the sample that underwent only the primary heat treatment. The curved lines are guides for the eye.

a continuous decrease in oxygen content. The primary sample, which was cooled to ambient temperature in 1 bar $\mathrm{O}_{2}$, was the most overdoped. When $T_{\max }$ was between $400{ }^{\circ} \mathrm{C}$ and $600{ }^{\circ} \mathrm{C}, T_{c}$ rose significantly from $\sim 86 \mathrm{~K}$ to $\sim 90 \mathrm{~K}$. Around $T_{\max }=800{ }^{\circ} \mathrm{C}$ the transition became very extended, suggesting that cation composition changes, not just oxygen doping.
Figure 2(c) shows Kramer function plots taken at $20 \mathrm{~K}$ with their linear extrapolation $H_{k}(20 \mathrm{~K})$ values given in Fig. 2(d). $H_{k}(20 \mathrm{~K})$ is initially improved by the $400{ }^{\circ} \mathrm{C}$ secondary HT compared to the primary HT, rising from 8.5 to $9.5 \mathrm{~T}$. However, after this initial increase, $H_{k}$ decreases monotonically with increasing secondary HT temperature. Using $H_{k}$ to approximate $H_{i r r}$, we interpret this decrease as a continuous 


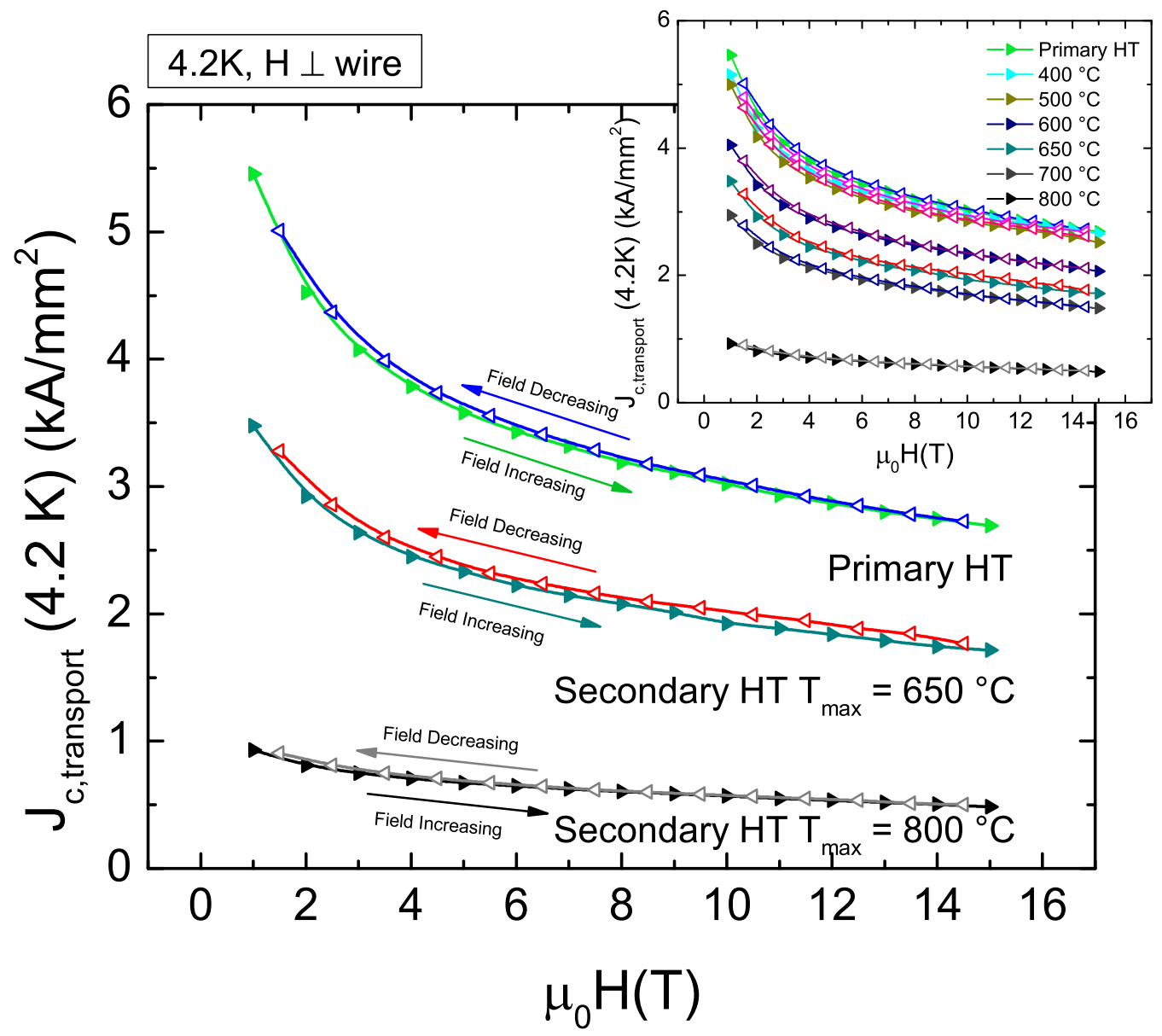

FIG. 3. Transport $J_{c}(4.2 \mathrm{~K})$ in increasing and decreasing perpendicular applied field for the samples secondary annealed at $650{ }^{\circ} \mathrm{C}$ and $800{ }^{\circ} \mathrm{C}$ compared to the primary heat treatment sample. The data for all samples are shown in the inset. $J_{c}(H)$ for samples secondary annealed at $T_{\max }=600{ }^{\circ} \mathrm{C}$ and higher are significantly smaller than for the optimally overdoped sample, yet none show any significant hysteresis.

decline in vortex pinning strength with an increasing electronic anisotropy induced by the decreasing carrier density [29,36].

Like $H_{k}$, the magnetization hysteresis $\Delta M(4.2 \mathrm{~K}, 5 \mathrm{~T})$ [Fig. 2(e)] for the $400{ }^{\circ} \mathrm{C}$ secondary HT samples showed a small increase above the primary HT samples but quickly declined with increasing secondary HT temperature. The trend is similar to that of transport $J_{c}$, as magnetization is proportional to the magnitude of currents circulating in the wire and the lengths over which these currents circulate [43].

Figure 2(f) plots transport $J_{c}(4.2 \mathrm{~K}, 15 \mathrm{~T})$, the most important performance metric for a superconducting wire. Transport $J_{c}$ follows a trend like that of $\Delta M(4.2 \mathrm{~K}, 5 \mathrm{~T})$, decreasing with increasing secondary annealing temperature. However, unlike $H_{k}$ and $\Delta M$, transport $J_{c}$ was not initially enhanced by a secondary HT, perhaps because the magnetization may have contributions from interfilament $\mathrm{Bi}-2212$ contacts that induce currents circulating on lengths shorter than the $1 \mathrm{~cm}$ gauge length of the transport measurements [43]. The fieldincreasing, field-decreasing behavior of transport $J_{c}$ is shown in Fig. 3. Strikingly, no sample exhibited significant $J_{c}(H)$ hysteresis, despite $J_{c}(H)$ values being much smaller for secondary HTs at $600{ }^{\circ} \mathrm{C}$ and higher. Transport $J_{c}$ hysteresis, present in even the best (and slightly overdoped) $\mathrm{Bi}-2223$ tapes, is a signature of GB weak links, where trapped flux penetrating weakly coupled GBs effectively reduces the applied field on the return path of the field, increasing the intergrain $J_{c}$ of the weak-link network over which the supercurrent flows $[3,44,45]$. The magnitude of $J_{c}$ in Fig. 2(f) is typical for wires made with Nexans granulate powder [2], about $2600 \mathrm{~A} \mathrm{~mm}^{-2}$ at $15 \mathrm{~T}, 4.2 \mathrm{~K}$. As discussed in the next section, more recent wires made with a finer, more uniform-sized powder have generated more than 2 times higher $J_{c}(4.2 \mathrm{~K}, 15 \mathrm{~T})$ with a tighter biaxial texture and with the same lack of hysteretic $J_{c}(H)$ characteristics.

Inverse pole figure maps (IPFs; Fig. 4) show the locally biaxial texture of colonies on the same longitudinal cross section of an individual $\mathrm{Bi}-2212$ filament in the highest- $J_{c}$ sample. Grains are colored with a mix of green, blue, and red according to the degree to which $\mathrm{Bi}-2212$ 's $[100] /[010]$, [110], and [001] crystallographic axes align with the projection axis. The projection axis is the filament axis in Fig. 4(a) and the direction perpendicular to both the filament axis and longitudinal cross section in Fig. 4(b). The black regions between and inside the grains represent Ag, voids, or secondary phases.

The typical grain size in Fig. 4 is $<1 \mu \mathrm{m}$ thick and $>40 \mu \mathrm{m}$ long, and the longest grains are mostly parallel to the filament 
(a)
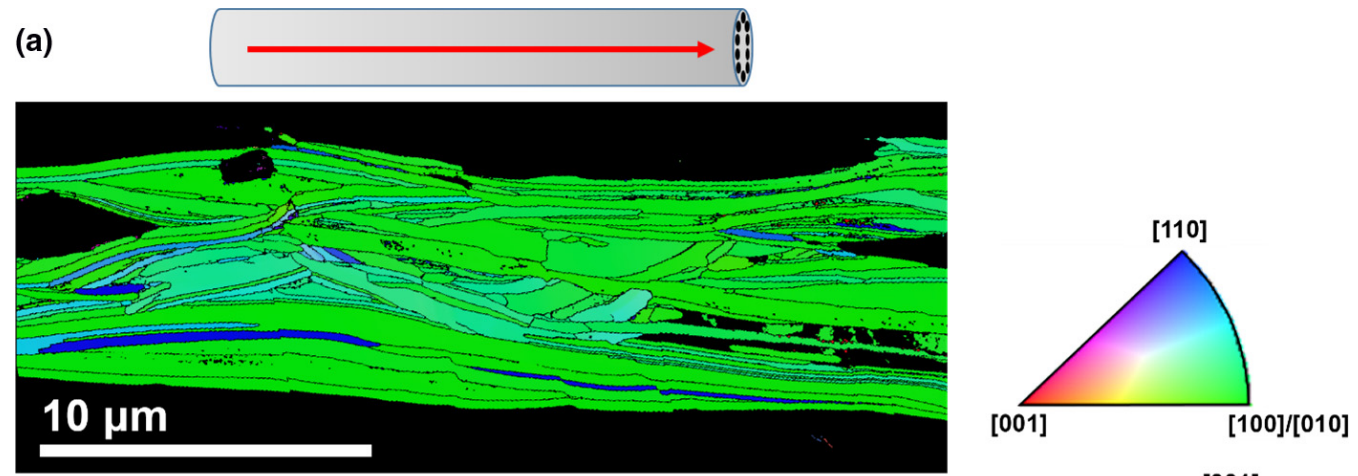

(b)
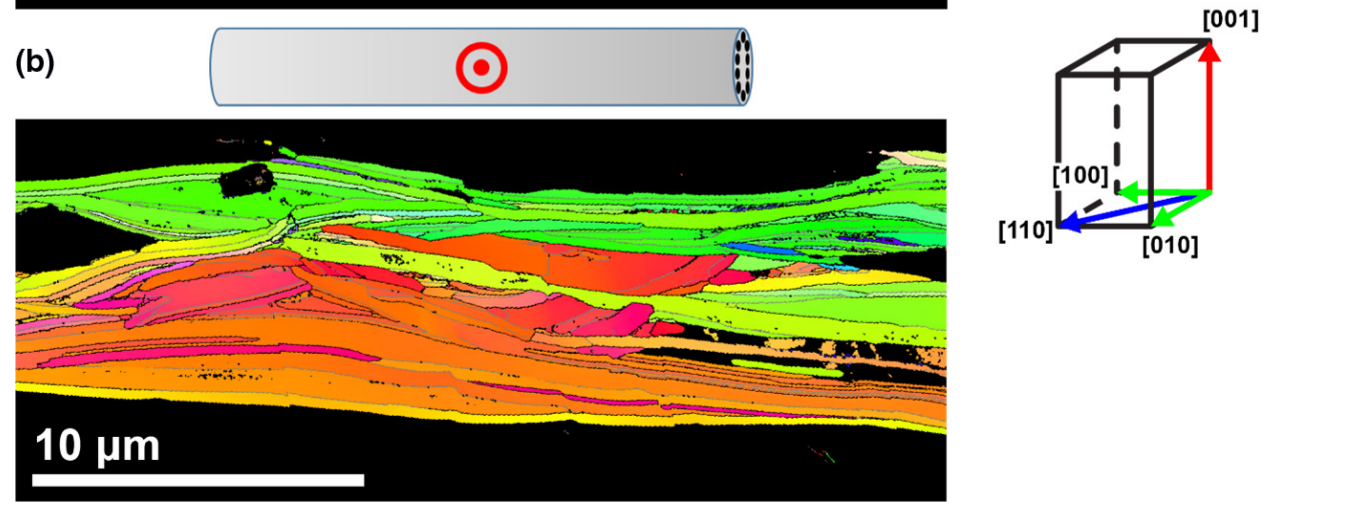

FIG. 4. Inverse pole figure maps of the same longitudinal cross section of an individual Bi-2212 filament in the highest- $J_{c}$ sample, colored according to the crystallographic triangle in the top right. Grains are colored with a mix of green, blue, and red according to the degree to which Bi-2212's [100]/[010], [110], and [001] crystallographic axes align with (a) the filament axis and (b) a direction normal to both the filament axis and the longitudinal cross section. As evidenced by the dominance of green in (a), the small filament diameter of $\sim 15 \mu \mathrm{m}$ constrains the fastest-growing $a$-axis grains to lie along the filament axis. As these grains grow, they tend to consume off-axis grains and plastically bend to smoothly align their basal planes together, generating a characteristic biaxial texture in which the $b$-axis [010] is perpendicular to the filament axis. Most grains are more than $40 \mu \mathrm{m}$ long, much longer than the grain thickness $(1 \mu \mathrm{m}$ maximum $)$ or the $15 \mu \mathrm{m}$ filament diameter. This large aspect ratio and the biaxial texture of individual colonies result in large basal-plane contact areas suitable for $c$-axis current flow around obstacles in the $a b$-plane current path. The black regions between and inside the grains represent Ag, voids or secondary phases.

axis. The large difference between the growth rate of $\mathrm{Bi}-2212$ grains along the $a$-axis [100] (fastest), $b$-axis [010] (slower), and $c$-axis [001] (slowest) results in thin, long platelike grains [10]. The small filament diameter of $\sim 15 \mu \mathrm{m}$ constrains the fast-growing $a$-axis grains to lie along the filament axis, where they can consume off-axis grains, thus generating a characteristic biaxial texture in which the $b$-axis [010] can lie in any plane perpendicular to the filament axis. EBSD cannot distinguish between the Bi-2212 $a$ and $b$ axes ([100] and [010], respectively) due to their almost identical lattice parameters. However, since grains grow the fastest along their $a$ axis and most grains in Fig. 4 are much longer along the filament axis, we deduce that green represents the $a$-axis [100] in Fig. 4(a) and the $b$-axis [010] in Fig. 4(b). This conclusion is well supported by the strong dominance of the color green in Fig. 4(a). In contrast, Fig. 4(b) shows two markedly different regions: the upper green area (close to [010]) and the lower red-/orange-colored grains (close to [001]), which indicates that $\mathrm{Bi}-2212$ grains tend to form colonies with shared [001] $(c$-axis) orientations. The $b$ axes of grains within these colonies azimuthally rotate about the filament axis. The two images together show that the filament cross section contains multiple biaxially textured colonies made up of long grains whose $a$ axes always lie along the filament direction. As a result, most of the basal-plane-faced grain boundaries are also parallel to the filament direction and [100] ( $a$ axis).

\section{DISCUSSION}

As noted earlier, extensive experiments and theoretical modeling of [001] tilt GBs between $a b$-plane-aligned grains, mostly for 123-YBCO [3-5] but also for 2212 [7] and 2223 [8,9] bicrystals, all agree that weak-link behavior starts at small misorientations of $5^{\circ}$ or less, a major cause of weak-link behavior being the lower hole and superfluid density of the GB compared to the grain. The depressed GB carrier density is often ascribed to ejection of $\mathrm{O}$ from the charge reservoir layers due to local GB or GB dislocation strains [46-49]. Even in the case of Ca-doped $R \mathrm{Ba}_{2} \mathrm{Cu}_{3} \mathrm{O}_{7-\delta}$ (RBCO) bicrystals, it is clear that the transport behavior of [001] tilt GBs improves near $T_{c}$ because $T_{c}$ depression caused by $\mathrm{Ca}$ addition is relieved by $\mathrm{Ca}$ desegregation away from the strongly coupled channels between the GB dislocations $[28,46,50]$. Thus, two observations of the present work are very significant:

(1) A $15^{\circ}$ FWHM biaxial texture clustered around [001], a misorientation well into the weak-link regime of [001] tilt bicrystal experiments, does not produce observable weak-link behavior. 
(2) Significantly underdoping Bi-2212 GBs in the current path does not produce observable weak-link behavior.

We therefore conclude that the local biaxial texture of $\mathrm{Bi}$ 2212 intrinsically constitutes a strongly coupled current path, regardless of its oxygen doping state. This is a quite unexpected result, incompatible with the standard explanation of current transport in polycrystalline HTS cuprates that strong links and weak links coexist in the active current path and that the path to higher $J_{c}$ is to ameliorate the doping state of the weak-link grain boundaries to one of higher carrier and superfluid density and, consequently, higher $J_{c}$.

There is a very important technological component to the present results. The very best uniaxially textured $\left(\sim 15^{\circ}\right.$ FWHM $)$ Bi-2223 tapes achieve $J_{c}(15 \mathrm{~T}, 4.2 \mathrm{~K})$ of $\sim 1100 \mathrm{~A} \mathrm{~mm}^{-2}$ with a hysteretic field-increasing/fielddecreasing weak-link characteristic, while these Bi-2212 round wires achieved $\sim 2600 \mathrm{~A} \mathrm{~mm}^{-2}$ and recent wires made with finer and more uniform powder [14] and similar processing can now achieve a $J_{c}(15 \mathrm{~T}, 4.2 \mathrm{~K})$ of $\sim 6500 \mathrm{~A} \mathrm{~mm}^{-2}$, all without weak-link hysteresis. Thus, Bi-2223 seems to depend on weak-link GBs for its high $J_{c}$ value, while Bi-2212 with 6 times higher $J_{c}$ does not. Moreover, round wire Bi-2212 has a completely flexible architecture with respect to number of filaments, wire and filament diameter, and $I_{c}$ rating and is also macroscopically isotropic and can be twisted to reduce $\mathrm{AC}$ loss [51]. By contrast, $\mathrm{Bi}-2223$ is anisotropic in shape and superconducting properties and made only in one size and one $I_{c}$ rating due to the complex reaction-deformation process that generates the uniaxial texture that maximizes its 6 times lower $J_{c}$. Yet, from an atomic structure point of view, Bi-2223 and $\mathrm{Bi}-2212$ are extremely close, sharing a common double $\mathrm{Bi}-\mathrm{O}$ charge reservoir layer and differing principally by the fact that $\mathrm{Bi}-2223$ has three $\mathrm{CuO}_{2}$ layers and $\mathrm{Bi}-2212$ has just two. In both respects, therefore, it seems that round wire $\mathrm{Bi}-2212$ breaks the paradigm that forces biaxially textured RBCO and uniaxially textured 2223 into tape geometries that reproduce the strong anisotropy of the native crystal structure and force expensive fabrication routes to ensure the best possible texture with minimum density of high-angle grain boundaries.

This paradigm-breaking behavior in $\mathrm{Bi}-2212$ can also be expressed in another way. Although its melt-textured biaxial texture is remarkable, its FWHM of $15^{\circ}$ is not, being far into the weak-link regime associated with [001] tilt bicrystals [10]. What [001] tilt bicrystals of many compositions show is that there is a hybrid misorientation regime in the $5^{\circ}-10^{\circ}$ range, where $J_{c}$ of parallel connected strongly coupled and weakly coupled portions of the GB is significantly degraded by withdrawing oxygen from the grain boundary [28,50,52]. It is precisely this behavior which is not seen here when we underdope $\mathrm{Bi}-2212$ round wires: while $J_{c}$ is reduced, there is no sign of former well-connected paths becoming weak linked.

We now should revisit the comparison of $\mathrm{Bi}-2212$ and $\mathrm{Bi}-$ 2223 in the context of the railway switch [24] and brick wall [22] models to explore how they might develop significantly different nonhysteretic and hysteretic $J_{c}(H)$ characteristics. It was clearly shown in Fig. 5 of the comparative Bi-2212/Bi2223 study of Kametani et al. [10] that Bi-2223 grains have essentially random $a b$-plane misorientations, while those of Bi-2212 are tightly clustered within about $15^{\circ}$ of [100]. Such
IPFs do not tell the whole story, however, as a further study by Kametani et al. on two recent $\mathrm{Bi}-2223$ tapes with $J_{c}$ values differing by $25 \%$ shows [15]. As in Ref. [10], many railway switch type intersections in which small-angle $c$-axis tilt grain boundaries obliquely butt into the basal plane of another are present in these $\mathrm{Bi}-2223$ tapes. Although not many smallangle $c$-axis tilt bicrystals have been studied, a few did fall into the angular range studied by Dimos et al. [4], where they were shown to be weakly linked for even small misorientations, consistent with the weak-link $J_{c}(H)$ hysteresis always measured in even the best $\mathrm{Bi}-2223$ tapes. By contrast $\mathrm{Bi}-2212$ grains tend to align their basal planes parallel to each other, often by plastic bending accommodation during the slow cooling from the melt stage of the heat treatment. The question of whether such basal-plane-faced grain boundaries would be weak links has, in fact, been studied by sintering single crystals together [53-56] and measuring the in-plane and $c$-axis transport $I_{c}$ properties. Very strikingly, it was found that $I_{c}$ was independent of [001] twist misorientation at the boundary and that no distinction could be made for $I_{c}$ measured with voltage taps along the $a b$ plane and across the grain boundary for $c$-axis current flow. Thus, there is some evidence that Bi-2212 [001] twist GBs connecting high aspect ratio grains, such as those found in textured tapes or in biaxially textured Bi-2212 round wires, can act as strongly coupled brick wall type connections limited by intragrain vortex pinning.

The $a b$-plane to $c$-axis grain aspect ratio is the foundation of the brick wall model. In Bulaevskii's model, the brick wall cartoon is of a standard brick with a $2: 1$ length to thickness ratio. But, as shown in the IPF in Fig. 4(a), Bi-2212 grains have a much more anisotropic shape, resulting in a length:thickness ratio of 40:1 or more. Figure 4(a), a longitudinal filament slice, clearly shows a strong [100] grain texture, in which the long dimension of aligned grains in the $a$-axis direction and their propensity to smoothly align their basal planes together is apparent. Most of the grains are more than $40 \mu \mathrm{m}$ long, much longer than the grain thickness ( $1 \mu \mathrm{m}$ maximum), which creates large basal-plane contact areas suitable for $c$-axis current flow around obstacles in the $a b$-plane current path, even though the $c$-axis $J_{c}$ is much lower than the $a b$-plane $J_{c}$. The strong $a$-axis texture means these anisotropic $\mathrm{Bi}-2212$ grains tend to stack parallel to each other, forming a large basal plane grain boundary area with only small misorientations that can favor $c$-axis transport. Bi-2212 grains can plastically bend during their high-temperature, slow-cooling step to minimize the strain between slightly misaligned grains, partially relieving the $c$-axis tilt misorientation and enhancing the basal-plane GB contact area. Thus, the dominant grain intersections in Bi-2212 are clearly the basal-plane faced [001] $c$-axis twist GBs of Bulaevskii et al. [22], despite the presence of some edge-on $c$-axis tilt or small-angle $c$-axis tilt grain boundaries that we expect from bicrystal studies to be weakly linked. The fact, therefore, that we do not observe weak-link hysteresis in $J_{c}(H)$ is consistent with the brick wall mode of supercurrent transport in $\mathrm{Bi}-2212$, where the $I_{c}$ of this strongly coupled brick wall type current path is limited by intragrain vortex pinning strength. We note that this strongly coupled current path occupies only a fraction of the superconducting volume, and we believe that recent advances in Bi-2212 round wire $J_{c}$ have come principally from increasing this fraction [13,57]. 


\section{SUMMARY}

We have made a careful study of the influence of underdoping on the superconducting properties of optimized Bi-2212 round wire superconductors. Very surprisingly, we found no evidence of weak-link behavior, even in the most underdoped state when both grains and grain boundaries are strongly underdoped. EBSD study of the grain structure shows that most grain-to-grain contacts occur by basal-plane-faced grain boundaries that earlier bicrystal studies have shown are not weakly linked. We conclude that modern Bi-2212 wires exemplify the non-weak-link brick wall model of supercurrent transport around obstacles in the current path, as opposed to the weak-link current path common in optimized $\mathrm{Bi}-2223$ tapes that has been attributed to railway switch transport. Moreover, round wire 2212 has recently doubled its $J_{c}$ values by using more uniform, finer particle powders. It thus seems that this method of developing brick wall current transport may still offer further opportunities to raise the critical current density of this most intriguing, round wire, macroscopically isotropic, and multifilament cuprate superconductor.

\section{ACKNOWLEDGMENTS}

The authors would like to thank V. Griffin and A. Francis for helping with and performing some of the transport $I_{c}$ and magnetization measurements and C. Tarantini for discussions and suggestions. This work was performed at the National High Magnetic Field Laboratory, which is supported by the NSF under Awards No. DMR-1157490 and No. DMR1644779 , and by the state of Florida. Primary support for this work was supplied by the U.S. DOE Office of High Energy Physics under Grant No. DE-SC0010421.
[1] H. Hilgenkamp and J. Mannhart, Grain boundaries in high- $T_{c}$ superconductors, Rev. Mod. Phys. 74, 485 (2002).

[2] D. C. Larbalestier, J. Jiang, U. P. Trociewitz, F. Kametani, C. Scheuerlein, M. Dalban-Canassy, M. Matras, P. Chen, N. C. Craig, P. J. Lee, and E. E. Hellstrom, Isotropic round-wire multifilament cuprate superconductor for generation of magnetic fields above 30 T, Nat. Mater. 13, 375 (2014).

[3] D. Dimos, P. Chaudhari, J. Mannhart, and F. K. LeGoues, Orientation Dependence of Grain-Boundary Critical Currents in $\mathrm{YBa}_{2} \mathrm{Cu}_{3} \mathrm{O}_{7-\delta}$ Bicrystals, Phys. Rev. Lett. 61, 219 (1988).

[4] D. Dimos, P. Chaudhari, and J. Mannhart, Superconducting transport properties of grain boundaries in $\mathrm{YBa}_{2} \mathrm{Cu}_{3} \mathrm{O}_{7}$ bicrystals, Phys. Rev. B 41, 4038 (1990).

[5] D. T. Verebelyi, D. K. Christen, R. Feenstra, C. Cantoni, A. Goyal, D. F. Lee, M. Paranthaman, P. N. Arendt, R. F. DePaula, J. R. Groves, and C. Prouteau, Low angle grain boundary transport in $\mathrm{YBa}_{2} \mathrm{Cu}_{3} \mathrm{O}_{7-\delta}$ coated conductors, Appl. Phys. Lett. 76, 1755 (2000).

[6] D. M. Feldmann, D. C. Larbalestier, D. T. Verebelyi, W. Zhang, Q. Li, G. N. Riley, R. Feenstra, A. Goyal, D. F. Lee, M. Paranthaman, D. M. Kroeger, and D. K. Christen, Inter- and intragrain transport measurements in $\mathrm{YBa}_{2} \mathrm{Cu}_{3} \mathrm{O}_{7-x}$ deformation textured coated conductors, Appl. Phys. Lett. 79, 3998 (2001).

[7] T. Amrein, L. Schultz, B. Kabius, and K. Urban, Orientation dependence of grain-boundary critical current densities in high$T_{c}$ bicrystals, Phys. Rev. B 51, 6792 (1995).

[8] A. Attenberger, J. Hänisch, B. Holzapfel, and L. Schultz, Electrical transport properties of Bi2223 [001] tilt grain boundary junctions, Physica C 372-376, 649 (2002).

[9] J. Hänisch, A. Attenberger, B. Holzapfel, and L. Schultz, Electrical transport properties of $\mathrm{Bi}_{2} \mathrm{Sr}_{2} \mathrm{Ca}_{2} \mathrm{Cu}_{3} \mathrm{O}_{10+\delta}$ thin film [001] tilt grain boundaries, Phys. Rev. B 65, 052507 (2002).

[10] F. Kametani, J. Jiang, M. Matras, D. Abraimov, E. E. Hellstrom, and D. C. Larbalestier, Comparison of growth texture in round Bi2212 and flat Bi2223 wires and its relation to high critical current density development, Sci. Rep. 5, 8285 (2015).

[11] T. Shen, J. Jiang, A. Yamamoto, U. Trociewitz, J. Schwartz, E. Hellstrom, and D. Larbalestier, Development of high critical current density in multifilamentary round-wire $\mathrm{Bi}_{2} \mathrm{Sr}_{2} \mathrm{CaCu}_{2} \mathrm{O}_{8+x}$ by strong overdoping, Appl. Phys. Lett. 95 , 152516 (2009).

[12] T. Shen, E. Bosque, D. Davis, J. Jiang, M. White, K. Zhang, H. Higley, M. Turqueti, Y. Huang, H. Miao, U. Trociewitz, E. Hellstrom, J. Parrell, A. Hunt, S. Gourlay, S. Prestemon, and D. C. Larbalestier, Stable, predictable and training-free operation of superconducting Bi-2212 Rutherford cable racetrack coils at the wire current density of $1000 \mathrm{~A} / \mathrm{mm}^{2}$, Sci. Rep. 9, 10170 (2019).

[13] M. D. Brown, J. Jiang, C. Tarantini, D. Abraimov, G. Bradford, J. Jaroszynski, E. E. Hellstrom, and D. C. Larbalestier, Prediction of the $J_{c}(\mathrm{~b})$ behavior of $\mathrm{Bi}-2212$ wires at high field, IEEE Trans. Appl. Supercond. 29, 6400504 (2019).

[14] J. Jiang, G. Bradford, S. I. Hossain, M. D. Brown, J. Cooper, E. Miller, Y. Huang, H. Miao, J. A. Parrell, M. White, A. Hunt, S. Sengupta, R. Revur, T. Shen, F. Kametani, U. P. Trociewitz, E. E. Hellstrom, and D. C. Larbalestier, High-performance Bi2212 round wires made with recent powders, IEEE Trans. Appl. Supercond. 29, 6400405 (2019).

[15] F. Kametani, T. A. Oloye, J. Jiang, G. Osabe, and S. Kobayashi, Visualization of the grain structure in the filament cross sections of uniaxially textured high $J_{c}$ Bi-2223 tapes, Appl. Phys. Express 12, 093002 (2019).

[16] K. Sato, Research, Fabrication and Applications of Bi-2223 HTS Wires (World Scientific, Singapore, 2016), Vol. 1.

[17] H. Rogalla and P. H. Kes, 100 Years of Superconductivity (CRC Press, Boca Raton, 2011).

[18] G. Wang, M. J. Raine, and D. P. Hampshire, The cause of 'weak-link' grain boundary behaviour in polycrystalline $\mathrm{Bi}_{2} \mathrm{Sr}_{2} \mathrm{Ca}_{1} \mathrm{Cu}_{2} \mathrm{O}_{8}$ and $\mathrm{Bi}_{2} \mathrm{Sr}_{2} \mathrm{Ca}_{3} \mathrm{Cu}_{3} \mathrm{O}_{10}$ superconductors, Supercond. Sci. Technol. 31, 024001 (2018).

[19] National Research Council, High Magnetic Field Science and Its Application in the United States: Current Status and Future Directions (National Academies Press, Washington, DC, 2013).

[20] H.-J. Liu, H.-J. Ma, F. Liu, Y. Shi, J.-G. Qin, Y. Wu, J.-G. Li, and L. Lei, Experimental study on Bi-2212 cable-in-conduit conductor, IEEE Trans. Appl. Supercond. 28, 4802104 (2018). 
[21] T. Shen, J. Jiang, F. Kametani, U. P. Trociewitz, D. C. Larbalestier, J. Schwartz, and E. E. Hellstrom, Filament to filament bridging and its influence on developing high critical current density in multifilamentary $\mathrm{Bi}_{2} \mathrm{Sr}_{2} \mathrm{CaCu}_{2} \mathrm{O}_{x}$ round wires, Supercond. Sci. Technol. 23, 025009 (2010).

[22] L. N. Bulaevskii, J. R. Clem, L. I. Glazman, and A. P. Malozemoff, Model for the low-temperature transport of Bibased high-temperature superconducting tapes, Phys. Rev. B 45, 2545 (1992)

[23] L. N. Bulaevskii, L. L. Daemen, M. P. Maley, and J. Y. Coulter, Limits to the critical current in high- $T_{c}$ superconducting tapes, Phys. Rev. B 48, 13798 (1993).

[24] B. Hensel, J. C. Grivel, A. Jeremie, A. Perin, A. Pollini, and R. Flükiger, $A$ model for the critical current in $(\mathrm{Bi}, \mathrm{Pb})_{2} \mathrm{Sr}_{2} \mathrm{Ca}_{2} \mathrm{Cu}_{3} \mathrm{O}_{x}$ silver-sheathed tapes, Physica C 205, 329 (1993).

[25] B. Hensel, G. Grasso, and R. Flükiger, Limits to the critical transport current in superconducting $(\mathrm{Bi}, \mathrm{Pb})_{2} \mathrm{Sr}_{2} \mathrm{Ca}_{2} \mathrm{Cu}_{3} \mathrm{O}_{10}$ silver-sheathed tapes: The railway-switch model, Phys. Rev. B 51, 15456 (1995).

[26] X. Y. Cai, A. Polyanskii, Q. Li, G. N. Riley, and D. C. Larbalestier, Current-limiting mechanisms in individual filaments extracted from superconducting tapes, Nature (London) 392, 906 (1998)

[27] M. Li, C. J. van der Beek, M. Konczykowski, A. A. Menovsky, and P. H. Kes, Superconducting properties of strongly underdoped $\mathrm{Bi}_{2} \mathrm{Sr}_{2} \mathrm{CaCu}_{2} \mathrm{O}_{8+x}$ single crystals, Phys. Rev. B 66, 024502 (2002).

[28] P. Li, D. Abraimov, A. Polyanskii, F. Kametani, and D. Larbalestier, Study of grain boundary transparency in YBCO bicrystal thin films over a wide temperature, field, and field orientation range, Phys. Rev. B 91, 104504 (2015).

[29] T. Nakane, A. Matsumoto, H. Kitaguchi, and H. Kumakura, Performance tuning and improvement of $J_{c}$-B characteristics for $\mathrm{Bi}-2212 / \mathrm{Ag}$ tapes by controlling the excess oxygen content, Supercond. Sci. Technol. 17, 29 (2004).

[30] T. Nakane, T. Mochiku, A. Matsumoto, H. Kitaguchi, and H. Kumakura, Effect of oxygen-pressure post-annealing on the $J_{c}$-B characteristics of Bi-2212/Ag tapes, Physica C 412-414, 1163 (2004).

[31] J. Shimoyama, Y. Nakayama, K. Kitazawa, K. Kishio, Z. Hiroi, I. Chong, and M. Takano, Strong flux pinning up to liquid nitrogen temperature discovered in heavily $\mathrm{Pb}$-doped and oxygen controlled Bi2212 single crystals, Physica C 281, 69 (1997).

[32] K. Kishio, J. Shimoyama, T. Kimura, Y. Kotaka, K. Kitazawa, K. Yamafuji, Q. Li, and M. Suenaga, Carrier doping and interlayer coupling in HTSC single crystals, Physica C 235, 2775 (1994).

[33] T. Nakane, M. Isobe, T. Mochiku, A. Matsumoto, H. Kitaguchi, and H. Kumakura, Effect of high oxygen pressure postannealing on the $J_{c}$-B characteristics of Bi-2212/Ag tapes, IEEE Trans. Appl. Supercond. 15, 2542 (2005).

[34] H. Kumakura, H. Kitaguchi, K. Togano, and N. Sugiyama, Effect of high oxygen partial pressure heat treatment on the superconducting properties of $\mathrm{Bi}_{2} \mathrm{Sr}_{2} \mathrm{CaCu}_{2} \mathrm{O}_{x} / \mathrm{Ag}$ tapes, J. Appl. Phys. 80, 5162 (1996).

[35] T. Haraguchi, T. Imada, M. Kiuchi, E. S. Otabe, T. Matsushita, T. Yasuda, S. Okayasu, S. Uchida, J. Shimoyama, and K. Kishio, Critical current density at low temperatures in $\mathrm{Bi}-2212$ superconductor with small anisotropy, Physica C 426-431, 304 (2005).

[36] V. F. Correa, E. E. Kaul, and G. Nieva, Overdoping effects in $\mathrm{Bi}_{2} \mathrm{Sr}_{2} \mathrm{CaCu}_{2} \mathrm{O}_{8+x}$ : From electromagnetic to Josephson interlayer coupling, Phys. Rev. B 63, 172505 (2001).

[37] A. Piriou, Y. Fasano, E. Giannini, and $\varnothing$. Fischer, Effect of oxygen-doping on $\mathrm{Bi}_{2} \mathrm{Sr}_{2} \mathrm{Ca}_{2} \mathrm{Cu}_{3} \mathrm{O}_{10+\delta}$ vortex matter: Crossover from electromagnetic to Josephson interlayer coupling, Phys. Rev. B 77, 184508 (2008).

[38] Y. Fukumoto, A. R. Moodenbaugh, M. Suenaga, D. A. Fischer, K. Shibutani, T. Hase, and S. Hayashi, Effect of oxygen partial pressure during postheat treatment on $\mathrm{Bi}_{2} \mathrm{Sr}_{2} \mathrm{CaCu}_{2} \mathrm{O}_{y} / \mathrm{Ag}$ tapes, J. Appl. Phys. 80, 331 (1996).

[39] I. Pallecchi, A. Leveratto, V. Braccini, V. Zunino, and A. Malagoli, Investigation of inter-grain critical current density in $\mathrm{Bi}_{2} \mathrm{Sr}_{2} \mathrm{CaCu}_{2} \mathrm{O}_{8+\delta}$ superconducting wires and its relationship with the heat treatment protocol, Supercond. Sci. Technol. 30, 095005 (2017).

[40] S. Yamashita, T. Kasai, T. Fujii, T. Watanabe, and A. Matsuda, Control of carrier concentration in Bi-2212, Physica C 470 , S170 (2010).

[41] M. Matras, J. Jiang, U. Trociewitz, D. Larbalestier, and E. Hellstrom, Process to densify $\mathrm{Bi}_{2} \mathrm{Sr}_{2} \mathrm{Ca}_{1} \mathrm{Cu}_{2} \mathrm{O}_{x}$ round wire with overpressure before coil winding and final overpressure heat treatment, Supercond. Sci. Technol. 33, 025010 (2020).

[42] E. J. Kramer, Scaling laws for flux pinning in hard superconductors, J. Appl. Phys. 44, 1360 (1973).

[43] M. A. Angadi, A. D. Caplin, J. R. Laverty, and Z. X. Shen, Nondestructive determination of the current-carrying length scale in superconducting crystals and thin films, Physica C 177, 479 (1991).

[44] A. I. D'yachenko, Hysteresis of transport critical current of high $\mathrm{T}_{c}$ superconductors in strong magnetic fields. Theory, Physica C 213, 167 (1993).

[45] J. E. Evetts and B. A. Glowacki, Relation of critical current irreversibility to trapped flux and microstructure in polycrystalline $\mathrm{YBa}_{2} \mathrm{Cu}_{3} \mathrm{O}_{7}$, Cryogenics 28, 641 (1988).

[46] X. Song, G. Daniels, D. M. Feldmann, A. Gurevich, and D. C. Larbalestier, Electromagnetic, atomic structure and chemistry changes induced by $\mathrm{Ca}$-doping of low-angle $\mathrm{YBa}_{2} \mathrm{Cu}_{3} \mathrm{O}_{7-\delta}$ grain boundaries, Nat. Mater. 4, 470 (2005).

[47] R. Klie, J. Buban, M. Varela, A. Franceschetti, C. Jooss, Y. Zhu, N. Browning, S. T. Pantelides, and S. J. Pennycook, Enhanced current transport at grain boundaries in high- $T_{c}$ superconductors, Nature (London) 435, 475 (2005).

[48] M. A. Schofield, M. Beleggia, Y. Zhu, K. Guth, and C. Jooss, Direct Evidence for Negative Grain Boundary Potential in Ca-Doped and Undoped $\mathrm{YBa}_{2} \mathrm{Cu}_{3} \mathrm{O}_{7-x}$, Phys. Rev. Lett. 92, 195502 (2004).

[49] A. Gurevich and E. A. Pashitskii, Current transport through low-angle grain boundaries in high-temperature superconductors, Phys. Rev. B 57, 13878 (1998).

[50] S.-I. Kim and D. C. Larbalestier, Influence of variable Cadoping on the critical current density of low-angle grain boundaries in $\mathrm{YBa}_{2} \mathrm{Cu}_{3} \mathrm{O}_{7-d}$, J. Appl. Phys. 128, 103905 (2020).

[51] Y. Oz, D. S. Davis, J. Jiang, E. E. Hellstrom, and D. C. Larbalestier, Influence of twist pitch on hysteretic losses and transport $J_{c}$ in overpressure processed high $J_{c}$ Bi-2212 round wires (unpublished). 
[52] S.-I. Kim and D. C. Larbalestier, Influence of strain-driven segregation in low-angle grain boundaries on critical current density in $\mathrm{Y}_{0.9} \mathrm{Nd}_{0.1} \mathrm{Ba}_{2} \mathrm{Cu}_{3} \mathrm{O}_{7-d}$, Supercond. Sci. Technol. 34, 025008 (2021).

[53] J. Wang, X. Cai, R. Kelley, M. Vaudin, S. Babcock, and D. Larbalestier, Electromagnetic coupling character of [001]-twist boundaries in sintered $\mathrm{Bi}_{2} \mathrm{Sr}_{2} \mathrm{CaCu}_{2} \mathrm{O}_{8+x}$ bicrystals, Physica $\mathrm{C}$ 230, 189 (1994)

[54] Q. Li, Y. N. Tsay, M. Suenaga, G. D. Gu, and N. Koshizuka, Superconducting coupling along the c-axis of [001] twist grainboundaries in $\mathrm{Bi}_{2} \mathrm{Sr}_{2} \mathrm{CaCu}_{2} \mathrm{O}_{8+\delta}$ bicrystals, Physica $\mathrm{C}$ 282, 1495 (1997).
[55] Y. Zhu, Q. Li, Y. N. Tsay, M. Suenaga, G. D. Gu, and N. Koshizuka, Structural origin of misorientation-independent superconducting behavior at [001] twist boundaries in Bi-2212, Phys. Rev. B 57, 8601 (1998).

[56] Q. Li, Y. N. Tsay, Y. Zhu, M. Suenaga, G. D. Gu, and N. Koshizuka, Electromagnetic and microstructural properties of bulk bicrystal grain boundaries in $\mathrm{Bi}_{2} \mathrm{Sr}_{2} \mathrm{CaCu}_{2} \mathrm{O}_{8+\delta}$ superconductors, Physica C 341-348, 1407 (2000).

[57] S. Barua, D. S. Davis, Y. Oz, J. Jiang, E. E. Hellstrom, U. P. Trociewitz, and D. C. Larbalestier, Critical current distributions of recent Bi-2212 round wires, IEEE Trans. Appl. Supercond. 31, 6400406 (2021). 